\title{
Neutrophil-to-lymphocyte ratio as a predictive marker for severe pediatric sepsis
}

\author{
Xi Zhong ${ }^{1}$, Aijia Ma ${ }^{1}$, Zhongwei Zhang ${ }^{1}$, Yong Liu' ${ }^{2}$, Guopeng Liang ${ }^{1}$ \\ ${ }^{1}$ Department of Critical Care Medicine, West China Hospital of Sichuan University, Chengdu, China; ${ }^{2}$ Department of Gastroenterological Surgery, \\ West China Hospital of Sichuan University, Chengdu, China \\ Contributions: (I) Conception and design: X Zhong, Y Liu, G Liang; (II) Administrative support: Y Liu, G Liang; (III) Provision of study materials \\ or patients: X Zhong, A Ma; (IV) Collection and assembly of data: X Zhong, A Ma; (V) Data analysis and interpretation: X Zhong, Z Zhang; \\ (VI) Manuscript writing: All authors; (VII) Final approval of manuscript: All authors. \\ Correspondence to: Guopeng Liang. Department of Critical Care Medicine, West China Hospital of Sichuan University, 37 Guoxue Lane, Wuhou \\ District, Chengdu, China. Email: HXLPG2021@163.com; Yong Liu. Department of Gastroenterological Surgery, West China Hospital of Sichuan \\ University, 37 Guoxue Lane, Wuhou District, Chengdu, China. Email: beckmanliuyong@163.com.
}

Background: Although neutrophil-to-lymphocyte ratio (NLR) has been extensively studied in several diseases, its role in pediatric sepsis remains unclear. Our study aimed to assess the predictive significance of NLR for severe pediatric sepsis in the pediatric intensive care unit (PICU).

Methods: We retrospectively recruited critically ill children in the PICU with severe pediatric sepsis from January 2019 to January 2020 in West China Hospital of Sichuan University. Univariate and multivariable logistic regression analysis was used to assess the risk factors of severe pediatric sepsis. Receiver operating characteristic (ROC) curves were plotted for the comparison of the prediction significance of NLR.

Results: Overall, 202 patients (severe sepsis 45; non-severe sepsis 157) were included. In the severe sepsis group, the levels of NLR $(\mathrm{P}<0.001)$, procalcitonin (PCT; $\mathrm{P}<0.001)$, and the Pediatric Risk of Mortality score (PRISM III) were higher than those in the nonsevere sepsis group $(\mathrm{P}<0.001)$. The $\mathrm{PICU}$ stay time $(\mathrm{P}<0.001)$, mechanical ventilation length $(\mathrm{P}=0.004)$, and hospital stay time $(\mathrm{P}<0.001)$ in the severe sepsis patients were noticeably more extended than those in the control patients. The area under the ROC curve (AUC) of NLR was $0.715(\mathrm{P}<0.001)$, which was higher than that of the PRISM III score (AUC $=0.651, \mathrm{P}<0.001)$ and PCT (AUC $=0.647, \mathrm{P}<0.001$ ). Furthermore, the constructed predictive model of NLR + PCT + PRISM III showed a better prediction significance than they alone (AUC $=0.888, \mathrm{P}<0.001$ ).

Conclusions: Results indicated that the initial NLR value was a significant biomarker for predicting severe pediatric sepsis. The combined NLR and PCT improved the evaluation for further early identification of severe sepsis in children.

Keywords: Critical care; pediatric sepsis; neutrophil-to-lymphocyte ratio (NLR); hematology

Submitted Jan 19, 2021. Accepted for publication Mar 05, 2021.

doi: $10.21037 / \mathrm{tp}-21-47$

View this article at: http://dx.doi.org/10.21037/tp-21-47

\section{Introduction}

Pediatric severe sepsis and septic shock continue to be a leading cause of pediatric intensive care unit (PICU) admissions, and have shown to be related to significant pediatric mortality and healthcare costs $(1,2)$. Alert and timely diagnosis and treatment continue to be recommended as the optimal method of choice for preventing severe pediatric sepsis and septic shock (3). Over the last decades, no single novel effective medical therapy or decisive diagnostic tool has been reported (3). Although the mortality of pediatric sepsis is declining, the burden of poor prognosis of severe sepsis in the pediatric population continues to be difficult to eliminate (4); therefore, the focus 
on this patient population should be intensified. Thus far, early the detection and treatment have been demonstrated to have the greatest effect in improving prognosis for pediatric severe sepsis patients.

Numerous biomarkers have been evaluated to distinguish sepsis from non infectious causes of SIRS, although none have been entirely useful. Procalcitonin (PCT) is currently the most frequently used biomarker for sepsis and increases during systemic inflammatory response and sepsis, especially in systemic bacterial, parasitic and fungal infections. However, the PCT testing is expensive and takes a long time. Therefore, clinicians need to develop tools which are inexpensive, rapid and easy to measure.

The neutrophils and the lymphocytes are the key cellular component of the human host defense system against an infection. During the course of sepsis, the number of white blood cells may vary, depending on the stage of sepsis, the patient's immunologic status, and the etiology of the infection. Increased number of neutrophils and decreased number of lymphocytes alert the clinician for an infection. A higher NLR may indicate a severe inflammatory progression in the patient (5). Although a number of clinical biomarkers for sepsis have been extensively studied, few have been examined in pediatric sepsis, especially severe sepsis (6-9). As a biomarker, the predictive and prognostic value of NLR in pulmonary and cardiovascular disease, as well as cancer, has been indicated $(10,11)$. There were many reports about the applications of neutrophil-to-lymphocyte ratio (NLR) as a predictive marker for adult sepsis, however, there was only one study about NLR for pediatric sepsis, and the included samples were few $(12,13)$. We enrolled 202 children to assess whether initial NLR at PICU admission could effectively predict the incidence of severe sepsis in children.

We present the following article in accordance with the STROBE reporting checklist (available at http://dx.doi. org/10.21037/tp-21-47).

\section{Methods}

\section{Study subjects}

This retrospective study recruited critically ill children with severe sepsis who were admitted to the PICU of the Department of Critical Care Medicine, West China Hospital of Sichuan University, from January 2019 to January 2020. The ethics committee of West China
Hospital of Sichuan University approved this study (No. NCT03598127). All procedures performed in this study involving human participants were in accordance with the declaration of Helsinki (as revised in 2013). Individual consent for this retrospective analysis was waived.

\section{Patient selection}

Patients were eligible for inclusion based on the following criteria: (I) older than 60 days and younger than 18 years; (II) diagnosed as severe sepsis or septic shock during PICU hospitalization; (III) counts of lymphocyte and neutrophil determined at PICU admission. Meanwhile, the exclusion criteria were as follows: (I) younger than 60 days or older than 18 years; (II) missing data regarding lymphocyte and neutrophil at PICU admission; (III) missing covariate data for multiple-variable alterations; (IV) repeated admissions to the PICU. Patients satisfying the recruitment standards were enrolled in the study.

\section{Definitions}

Severe sepsis was defined according to the criteria established by the International Pediatric Sepsis Consensus Conference (14). Severe sepsis was considered present when the patient met one of the following criteria: (I) cardiovascular system dysfunction, (II) acute respiratory distress syndrome, (III) dysfunction of 2 or more other organs (cardiovascular, respiratory, neurologic, hematologic, renal, hepatic). Septic shock was defined as sepsis and cardiovascular organ dysfunction. In our study, we included septic shock in severe sepsis.

\section{Data collection and outcomes}

We collected the following data for all the patients within 24 hours of admission from the database at PICU: demographic information, source of infection, and laboratory tests. To estimate disease severity, Pediatric Risk of Mortality (PRISM III) score, a physiology-based predictor for risk of mortality, was calculated at the baseline within 24 hours of admission at the PICU (15). We collected laboratory findings, including white blood cell, neutrophil, lymphocyte, monocyte, and platelet counts; NLR and platelet-to-lymphocyte ratio (PLR); and albumin and PCT levels. The primary outcome was all-cause mortality 28 days after PICU arrival. Secondary clinical 


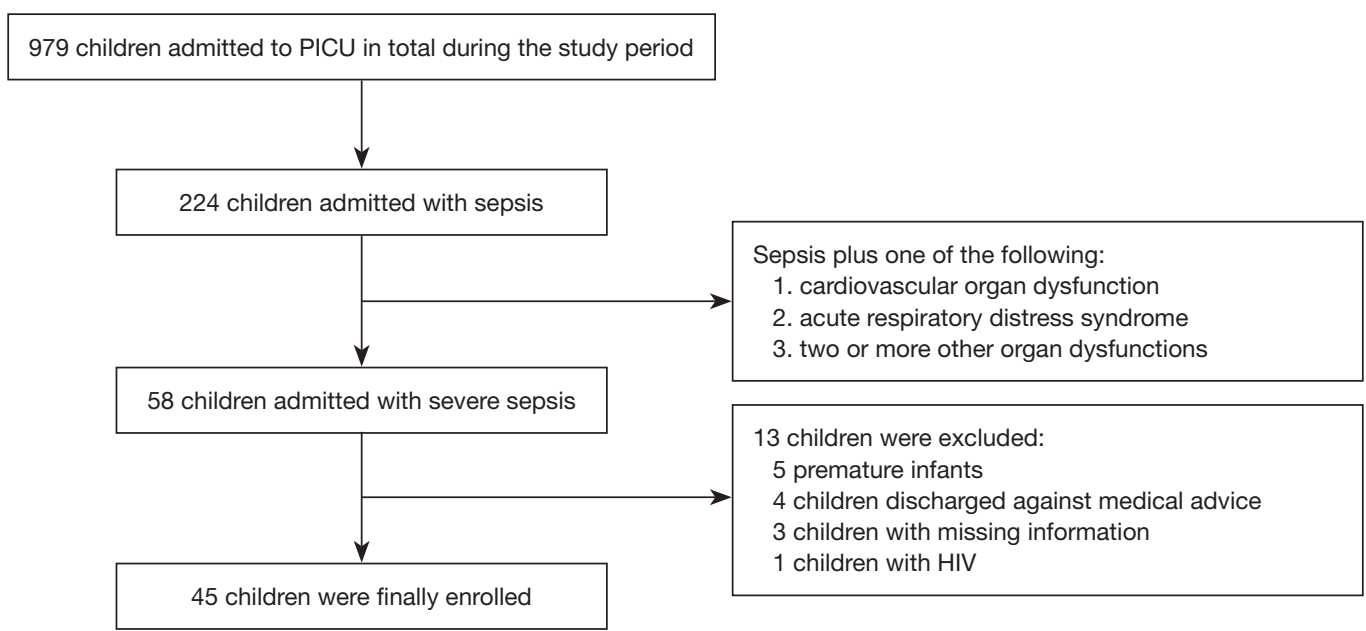

Figure 1 The flow chart of screening and enrollment. PICU, pediatric intensive care unit.

outcomes were hospital stay time, PICU stay time, and mechanical ventilation length.

\section{Statistical analysis}

SPSS version 22.0 (IBM Corp., USA) and MedCalc version 15.0 were used for statistical analyses. General distribution data are expressed as mean and SD, while binomial variables are expressed as median or interquartile range (IQR). Categorical variables were compared using the $\chi^{2}$ test. The variables of the severe sepsis and control groups were compared using the $t$-test or Mann-Whitney test. Univariate and subsequent multivariate logistic regression analyses were used to assess risk factors of severe pediatric sepsis.

Area under the receiver operating characteristic curve (AUC) was used to evaluate the variables' predictive performance. When a significant AUC was generated, the maximum possible sum of the sensitivity and specificity levels was considered to be the optimal cutoff point. A $\mathrm{P}$ value $<0.05$ indicated statistical significance.

\section{Results}

\section{Baseline characteristics of the patient}

During the study period, a total of 202 patients (severe sepsis 45, non-severe sepsis 157) were enrolled in the final analysis (Figure 1). Among all 45 severe sepsis patients, 8 patients $(17.78 \%)$ had septic shock. The median age was 13 months (IQR 3.00-78.00), and 115 (56.9\%) patients were male. The median PRISM III score was 10 (IQR 6.0015.00). The PRISM III score of severe pediatric sepsis was higher compared to the control group $(\mathrm{P}=0.002)$. Moreover, of the total patients, $17.8 \%$ had respiratory system infection, $41.1 \%$ had gastrointestinal system infection, 5.4\% had central nervous system infection, $5.0 \%$ had bloodstream infection, and $12.9 \%$ had multiple infections. The number of multiple infections in the severe sepsis group was noticeably higher than that in the nonsevere sepsis group $(\mathrm{P}<0.001)$.

In the laboratory tests, the median value of PCT was 2.21 (IQR 0.37-11.82) and NLR was 3.73 (IQR 2.04-6.59). The NLR and PCT levels were significantly higher in the severe sepsis group compared to the control group $(\mathrm{P}<0.001$, $\mathrm{P}=0.003$, respectively). Finally, the length of PICU stay time $(\mathrm{P}<0.001)$, hospital stay time $(\mathrm{P}<0.001)$, and mechanical ventilation time $(\mathrm{P}=0.004)$ in the severe sepsis group were longer than those in the control group, but no statistical significance was found in 28-day mortality $(\mathrm{P}=0.066)$. The baseline clinical and laboratory features of the patients are described in Table 1.

\section{Logistic regression analysis}

Univariate logistic regression was conducted to determine the relationships of the respective variables with the incidence of severe sepsis. The results indicated that age $(\mathrm{P}=0.004)$, weight $(\mathrm{P}=0.012)$, multiple infections $(\mathrm{P}=0.001)$, NLR $(\mathrm{P}<0.001)$, PCT $(\mathrm{P}<0.001)$, and PRISM III $(\mathrm{P}<0.001)$ were risk factors of severe sepsis. In multivariate logistic regression analysis, multiple infections ( $\mathrm{P}=0.013)$, NLR $(\mathrm{P}=0.006)$, PCT 
Table 1 Baseline characteristics

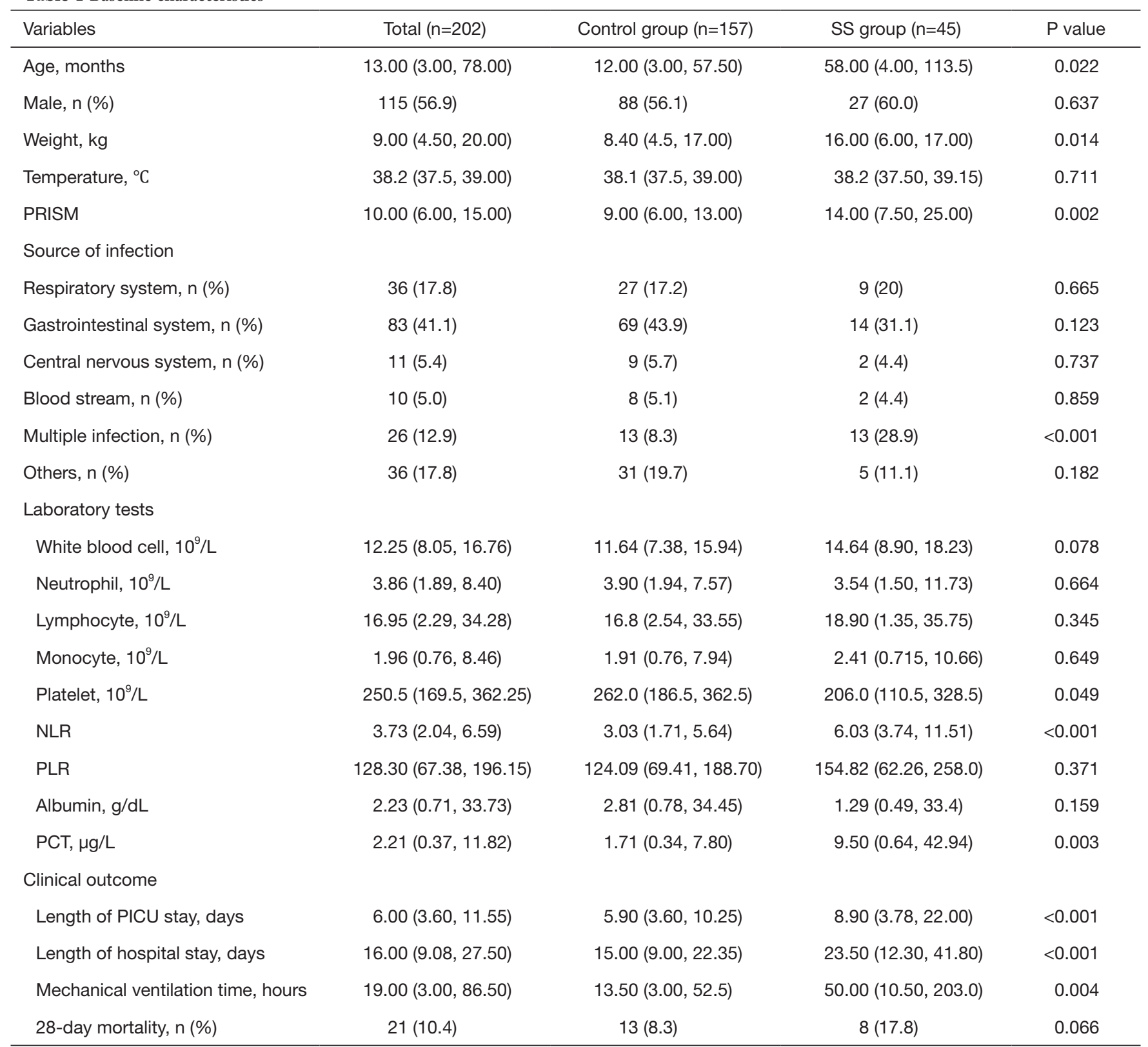

All values are expressed as $\mathrm{n}$ (\%) or median (first to third quartile). SS group, severe sepsis group; PRISM, Pediatric Risk of Mortality; NLR, neutrophil-to-lymphocyte ratio; PLR, platelet-to-lymphocyte ratio; PCT, procalcitonin; PICU, pediatric intensive care unit.

$(\mathrm{P}=0.025)$, and PRISM III $(\mathrm{P}<0.001)$ were associated with the incidence of severe sepsis. The results of univariate and multivariate logistic regression are shown in Table 2.

\section{Predictive value of NLR}

The AUC, cutoff value, sensitivity, specificity of PRISM III, PCT, and NLR for predicting severe sepsis are presented in Table 3. NLR had the highest predictive power for severe sepsis with an AUC of 0.715 and a cutoff value of 4.06; this was followed by PRISM III score (AUC $=0.651, \mathrm{P}<0.001$ ) and $\mathrm{PCT}(\mathrm{AUC}=0.647, \mathrm{P}<0.001)$ (Figure 2).

\section{Predictive value of the constructed model}

We constructed a combined Receiver operating characteristic 
Table 2 Univariate analysis and multivariate logistic regression analysis of risk factors associated with pediatric severe sepsis

\begin{tabular}{|c|c|c|c|c|}
\hline Variables & \multicolumn{2}{|c|}{ Univariate analysis } & \multicolumn{2}{|c|}{ Multivariate analysis } \\
\hline Age & $1.008(1.003,1.014)$ & 0.004 & $0.994(0.974,1.014)$ & 0.552 \\
\hline Male & $1.176(0.599,2.309)$ & 0.637 & $0.716(0.261,1.967)$ & 0.517 \\
\hline Weight & $1.032(1.007,1.058)$ & 0.012 & $1.035(0.950,1.128)$ & 0.43 \\
\hline Gastrointestinal system & $0.576(0.284,1.166)$ & 0.125 & $0.612(0.129,2.903)$ & 0.536 \\
\hline Central nervous system & $0.765(0.159,3.674)$ & 0.738 & $0.449(0.036,5.607)$ & 0.534 \\
\hline Blood stream & $0.866(0.177,4.232)$ & 0.859 & $2.672(0.107,66.699)$ & 0.549 \\
\hline Multiple infection & $4.50(1.906,10.623)$ & 0.001 & $8.084(1.559,41.927)$ & 0.013 \\
\hline Lymphocyte & $0.998(0.978,1.018)$ & 0.861 & $0.967(0.897,1.043)$ & 0.386 \\
\hline Monocyte & $1.034(0.975,1.096)$ & 0.265 & $0.881(0.674,1.153)$ & 0.357 \\
\hline Platelet & $0.999(0.996,1.001)$ & 0.222 & $1.001(0.997,1.006)$ & 0.619 \\
\hline NLR & $1.143(1.062,1.230)$ & $<0.001$ & $1.259(1.070,1.481)$ & 0.006 \\
\hline PLR & $1.003(1.000,1.005)$ & 0.036 & $0.997(0.989,1.004)$ & 0.392 \\
\hline Albumin & $0.994(0.975,1.013)$ & 0.548 & $0.962(0.896,1.034)$ & 0.296 \\
\hline РCT & $1.022(1.01,1.034)$ & $<0.001$ & $1.018(1.002,1.035)$ & 0.025 \\
\hline Temperature & $1.077(0.766,1.514)$ & 0.671 & $0.754(0.439,1.298)$ & 0.309 \\
\hline
\end{tabular}

NLR, neutrophil-to-lymphocyte ratio; PLR, platelet-to-lymphocyte ratio; PCT, procalcitonin; PRISM, Pediatric Risk of Mortality; OR, odds ratio; $\mathrm{Cl}$, confidence interval.

Table 3 Comparison of AUC values for predicting pediatric severe sepsis

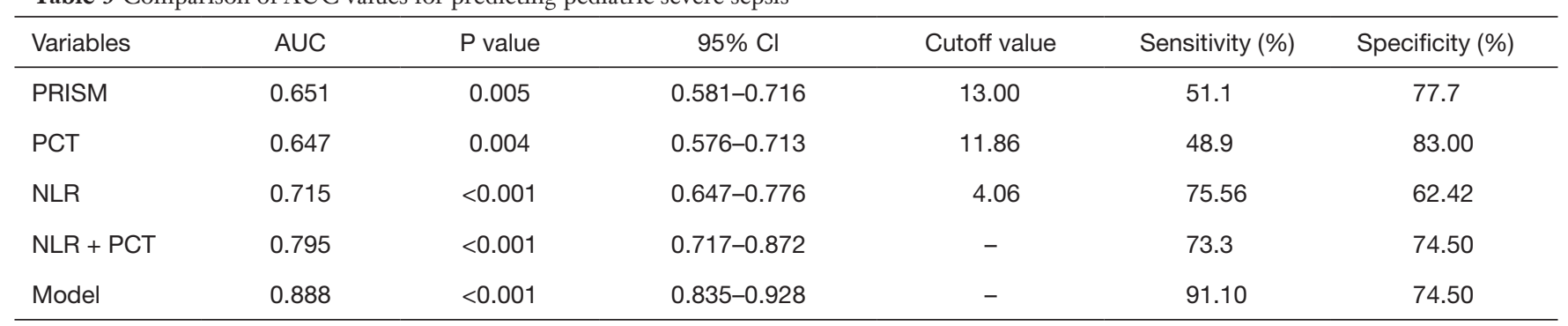

AUC, area under the receiver operating characteristic curve; PRISM, Pediatric Risk of Mortality; NLR, neutrophil-to-lymphocyte ratio; PCT, procalcitonin; $\mathrm{Cl}$, confidence interval.

(ROC) curve model of NLR and PCT. The AUC of the NLR + PCT model was $0.795(\mathrm{P}<0.001)$, which was noticeably higher than that of the NLR-alone $(Z=2.051$, $\mathrm{P}=0.043)$ and PCT-alone $(\mathrm{Z}=3.108, \mathrm{P}=0.002)$ models
(Figure 2). Furthermore, we made use of three statistically significant factors, including PRISM III, PCT, and NLR, to construct the predictive model. The predictive model of NLR+PCT+PRISM III exhibited an AUC of 0.888 
$(\mathrm{P}<0.001)$. The sensitivities and specificities of the predictive model were $91.1 \%$ and $74.5 \%$, respectively, which were noticeably higher than those of the NLR + PCT $(Z=2.948$,

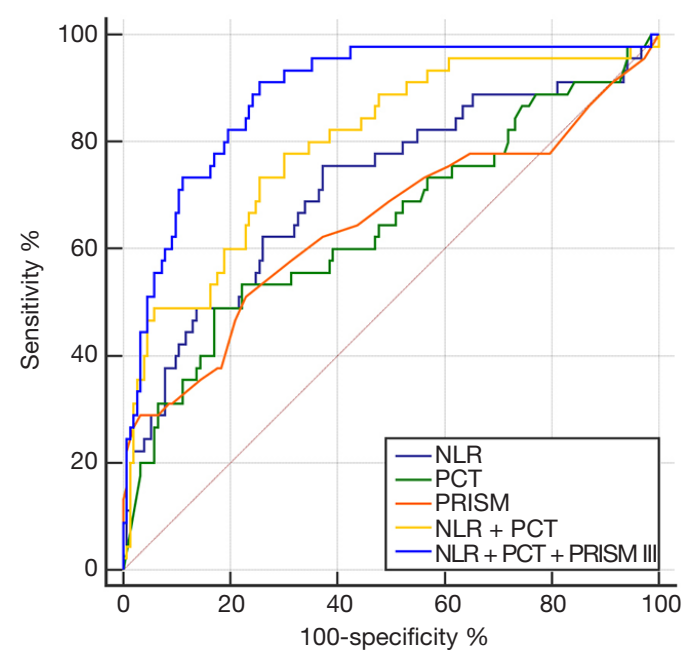

Figure 2 ROC curve analysis comparing the predictive value of severe pediatric sepsis. ROC, receiver operating characteristic curve.
$\mathrm{P}=0.003$ ) model (Figure 2). The nomogram and calibration plot for NLR + PCT in predicting severe sepsis in pediatric patients are presented in Figure 3.

\section{Discussion}

In this study, we found that the initial NLR obtained at PICU admission was a significant predictive biomarker for pediatric severe sepsis patients. Moreover, the predictive model of NLR + PCT + PRISM III demonstrated a greater prediction performance and could provide earlier detection of severe sepsis and better guide clinical practice. Our research placed a higher emphasis on assessing the incidence of severe sepsis in children, which might be the key to reducing mortality in the PICU. This study was the first to examine the relationship between NLR and severe pediatric sepsis in the PICU.

Unlike sepsis, severe sepsis is more associated with the failure of multiple organs and includes cardiovascular, respiratory, neurologic, hematologic, renal, and hepatic dysfunction (14). Mortality for severe pediatric sepsis has been reported to be nearly 3 times higher than that for regular sepsis (16). Thus, severe sepsis is a key
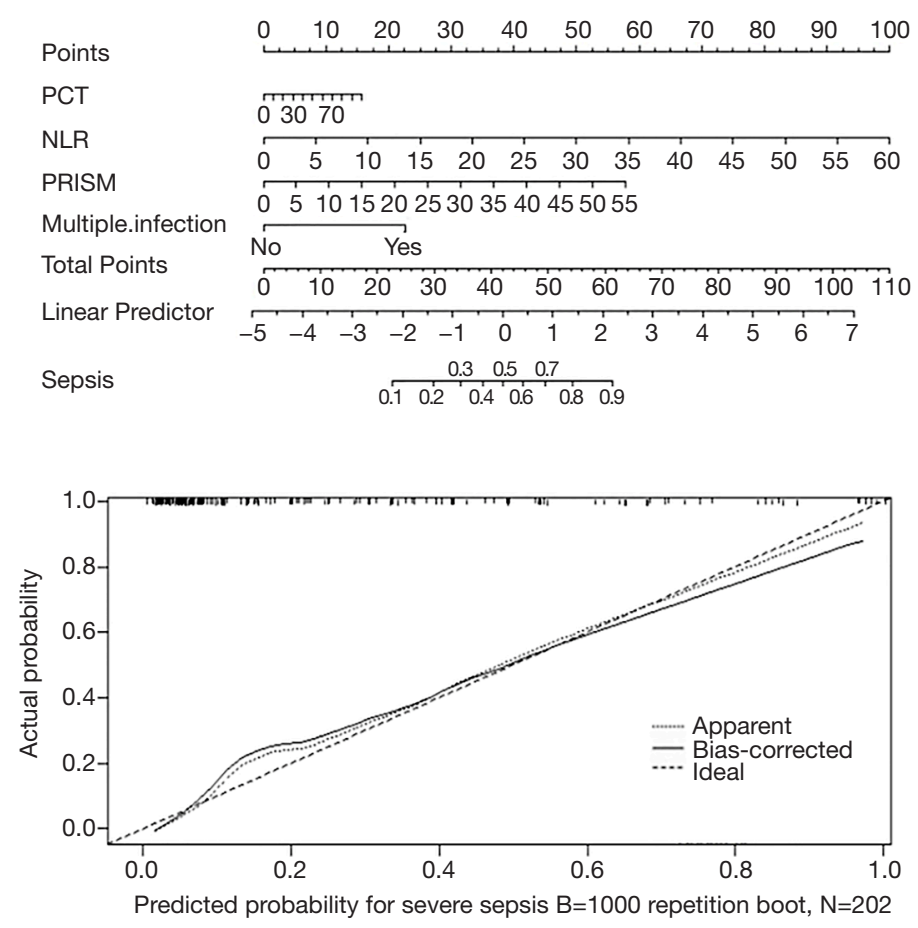

Figure 3 NLR + PCT in predicting severe sepsis in pediatric patients. (A) Nomogram of the constructed predictive model for predicting severe sepsis in included pediatric patients. (B) Calibration plot of the constructed predictive model for predicting severe sepsis in included pediatric patients. 
contributor to high ICU mortality in children, and our study has highlighted the need for the early detection of this condition. Before severe sepsis develops into septic shock, early identification of patients who may progress to severe sepsis and antibiotic treatment and efficient fluid resuscitation may reduce mortality (17-20).

The causes of elevated NLR and poor prognosis in severe sepsis patients are unclear. One of the most convincing explanations is primarily based on the physiological link between systemic inflammation and stress (21-24). NLR is able to indicate the patient's response to inflammation. The upregulation in the number of neutrophils may indicate the presence of an uncleared infected lesion which inhibits lymphocytes. Accumulating evidence has pointed to neutrophils as vital cellular components of the natural immune system's defense against infection, with lymphocytes the being main cellular component of the adaptive immune system $(25,26)$. Lymphocytes play an essential role in regulating inflammation, and the loss of lymphocytes attributed to apoptosis induced by persistent sepsis is likely to cause the suppression of the immune system (26). Therefore, neutropenia and lymphopenia resulting from the continuous suppression of the immune system may be the theoretical basis underlying the ability of NLR to predict severe sepsis.

In the present study, the predictive significance of NLR, combined with PCT and PRISM III score, in pediatric severe sepsis patients in the PICU was comparatively assessed. NLR was revealed to have a significant predictive capacity to predict severe sepsis in comparison with PCT in pediatric patients. Furthermore, the constructed predictive model of NLR + PCT + PRISM III showed an even better prediction significance than that of each of these factors used alone. The PRISM III score (15) was assessed used 17 physiologic variables classified in the following fashion: (I) cardiovascular and nervous system (systolic blood pressure, heart rate, temperature, Glasgow coma scale, pupillary reactions); (II) blood gas (acidosis, $\mathrm{CO}_{2}, \mathrm{pH}, \mathrm{PaCO}_{2}, \mathrm{PaO}_{2}$ ); (III) blood routine and biochemistry test (white blood cell count, platelet count, partial thromboplastin time). As can be seen in the variables above, there is little emphasis to no emphasis on inflammation, neutrophils, and lymphocytes in the blood routine for calculating the score. Therefore, the addition of NLR and PCT can enhance the capacity of the PRISM III scores to predict severe sepsis.

Studies have shown that C-reactive protein (CRP) and PCT are good indicators for predicting sepsis in children (6). However, under various inflammatory conditions, CRP and
PCT levels can also be elevated, so there is limited value in identifying infection from other causes of inflammation. Furthermore, the cost of PCT and CRP testing is relatively higher than that of NLR, especially CRP. Since there is no apparent inflammatory reaction in the early sepsis stage, PCT and CRP are not used as routine testing items. Thus, NLR also possesses the advantage of being more economical for early prediction.

This study has a few limitations that should be considered. First, our study was limited by its retrospective design. Second, because our hospital did not routinely detect CRP values, the authors could not compare the predictive ability of NLR and CRP. We hope that further research will focus on this aspect. Third, the number of patients enrolled in the study was small, and prospective analyses based on larger sample sizes are required for indepth verification.

\section{Conclusions}

Among NLR, PRISM III, and PCT, the initial NLR value at PICU admission was the most useful biomarker for predication of severe sepsis in pediatric patients. The constructed predictive model of NLR + PCT + PRISM III was a highly sensitive and specific predictive model for severe sepsis, demonstrating great potential for greater prediction accuracy of severe sepsis in children.

\section{Acknowledgments}

We acknowledge all staff who offered help for this study and particularly acknowledge Qiao Wang, from the Department of Medical Statistics in Sichuan University, for providing advice regarding the data analysis.

Funding: This work was supported by the Provincial Science and Technology Department Project in Sichuan (No. 2020YFS0164).

\section{Footnote}

Reporting Checklist: The authors have completed the STROBE reporting checklist. Available at http://dx.doi. org/10.21037/tp-21-47

Data Sharing Statement: Available at http://dx.doi. org/10.21037/tp-21-47

Conflicts of Interest: All authors have completed the ICMJE 
uniform disclosure form (available at http://dx.doi. org/10.21037/tp-21-47). The authors have no conflicts of interest to declare.

Ethical Statement: The authors are accountable for all aspects of the work in ensuring that questions related to the accuracy or integrity of any part of the work are appropriately investigated and resolved. The ethics committee of West China Hospital of Sichuan University approved this study (No. NCT03598127). All procedures performed in this study involving human participants were in accordance with the declaration of Helsinki (as revised in 2013). Individual consent for this retrospective analysis was waived.

Open Access Statement: This is an Open Access article distributed in accordance with the Creative Commons Attribution-NonCommercial-NoDerivs 4.0 International License (CC BY-NC-ND 4.0), which permits the noncommercial replication and distribution of the article with the strict proviso that no changes or edits are made and the original work is properly cited (including links to both the formal publication through the relevant DOI and the license). See: https://creativecommons.org/licenses/by-nc-nd/4.0/.

\section{References}

1. Tan B, Wong JJ, Sultana R, et al. Global Case-Fatality Rates in Pediatric Severe Sepsis and Septic Shock: A Systematic Review and Meta-analysis. JAMA Pediatr 2019;173:352-62.

2. Salciccioli JD, Marshall DC, Pimentel MA, et al. The association between the neutrophil-to-lymphocyte ratio and mortality in critical illness: an observational cohort study. Crit Care 2015;19:13.

3. Fleischmann-Struzek C, Goldfarb DM, Schlattmann P, et al. The global burden of paediatric and neonatal sepsis: a systematic review. Lancet Respir Med 2018;6:223-30.

4. Balamuth F, Weiss SL, Neuman MI, et al. Pediatric severe sepsis in U.S. children's hospitals. Pediatr Crit Care Med 2014;15:798-805.

5. Liu Y, Zheng J, Zhang D, et al. Neutrophil-lymphocyte ratio and plasma lactate predict 28-day mortality in patients with sepsis. J Clin Lab Anal 2019;33:e22942.

6. Ryu JA, Yang JH, Lee D, et al. Clinical Usefulness of Procalcitonin and C-Reactive Protein as Outcome Predictors in Critically Ill Patients with Severe Sepsis and Septic Shock. PLoS One 2015;10:e0138150.
7. Yu MH, Chen MH, Han F, et al. Prognostic value of the biomarkers serum amyloid $\mathrm{A}$ and nitric oxide in patients with sepsis. Int Immunopharmacol 2018;62:287-92.

8. Behnes M, Bertsch T, Lepiorz D, et al. Diagnostic and prognostic utility of soluble CD 14 subtype (presepsin) for severe sepsis and septic shock during the first week of intensive care treatment. Crit Care 2014;18:507.

9. Sarı R, Karakurt Z, Ay M, et al. Neutrophil to lymphocyte ratio as a predictor of treatment response and mortality in septic shock patients in the intensive care unit. Turk J Med Sci 2019;49:1336-49.

10. Templeton AJ, McNamara MG, Seruga B, et al. Prognostic role of neutrophil-to-lymphocyte ratio in solid tumors: a systematic review and meta-analysis. J Natl Cancer Inst 2014;106:dju124.

11. Lattanzi S, Brigo F, Trinka E, et al. Neutrophil-toLymphocyte Ratio in Acute Cerebral Hemorrhage: a System Review. Transl Stroke Res 2019;10:137-45.

12. Diem S, Schmid S, Krapf M, et al. Neutrophil-toLymphocyte ratio (NLR) and Platelet-to-Lymphocyte ratio (PLR) as prognostic markers in patients with nonsmall cell lung cancer (NSCLC) treated with nivolumab. Lung Cancer 2017;111:176-81.

13. Ayca B, Akin F, Celik O, et al. Neutrophil to Lymphocyte Ratio is Related to Stent Thrombosis and High Mortality in Patients With Acute Myocardial Infarction. Angiology 2015;66:545-52.

14. Goldstein B, Giroir B, Randolph A, et al. International pediatric sepsis consensus conference: definitions for sepsis and organ dysfunction in pediatrics. Pediatr Crit Care Med 2005;6:2-8.

15. Pollack MM, Holubkov R, Funai T, et al. The Pediatric Risk of Mortality Score: Update 2015. Pediatr Crit Care Med 2016;17:2-9.

16. Weiss SL, Fitzgerald JC, Pappachan J, et al. Global epidemiology of pediatric severe sepsis: the sepsis prevalence, outcomes, and therapies study. Am J Respir Crit Care Med 2015;191:1147-57.

17. Workman JK, Ames SG, Reeder RW, et al. Treatment of Pediatric Septic Shock With the Surviving Sepsis Campaign Guidelines and PICU Patient Outcomes. Pediatr Crit Care Med 2016;17:e451-8.

18. Atreya MR, Wong HR. Precision medicine in pediatric sepsis. Curr Opin Pediatr 2019;31:322-7.

19. Paul R. Recognition, Diagnostics, and Management of Pediatric Severe Sepsis and Septic Shock in the Emergency Department. Pediatr Clin North Am 2018;65:1107-18.

20. Aygün F, Varol F, Durak C, et al. Evaluation of Continuous 
Renal Replacement Therapy and Therapeutic Plasma Exchange, in Severe Sepsis or Septic Shock in Critically Ill Children. Medicina (Kaunas) 2019;55:350.

21. Westerdijk K, Simons KS, Zegers M, et al. The value of the neutrophil-lymphocyte count ratio in the diagnosis of sepsis in patients admitted to the Intensive Care Unit: A retrospective cohort study. PLoS One 2019;14:e0212861.

22. Delano MJ, Ward PA. The immune system's role in sepsis progression, resolution, and long-term outcome. Immunol Rev 2016;274:330-53.

23. Hotchkiss RS, Monneret G, Payen D. Sepsis-induced immunosuppression: from cellular dysfunctions to

Cite this article as: Zhong X, Ma A, Zhang Z, Liu Y, Liang G. Neutrophil-to-lymphocyte ratio as a predictive marker for severe pediatric sepsis. Transl Pediatr 2021;10(3):657-665. doi: $10.21037 /$ tp-21-47 immunotherapy. Nat Rev Immunol 2013;13:862-74.

24. Chousterman BG, Swirski FK, Weber GF. Cytokine storm and sepsis disease pathogenesis. Semin Immunopathol 2017;39:517-28.

25. Zahorec R. Ratio of neutrophil to lymphocyte counts-rapid and simple parameter of systemic inflammation and stress in critically ill. Bratisl Lek Listy 2001;102:5-14.

26. Heffernan DS, Monaghan SF, Thakkar RK, et al. Failure to normalize lymphopenia following trauma is associated with increased mortality, independent of the leukocytosis pattern. Crit Care 2012;16:R12. 